\title{
The osteoarthritis-associated gene PAPSS2 promotes differentiation and matrix formation in ATDC5 chondrogenic cells
}

\author{
LIYING FAN ${ }^{1}$, YUAN HE $^{2}$, JING HAN ${ }^{3}$, PUWEI YUAN ${ }^{4}$, XIONG GUO $^{3}$ and WEIZHUO WANG ${ }^{1}$ \\ ${ }^{1}$ Department of Orthopedic Surgery, The Second Affiliated Hospital, Xi'an Jiaotong University, Xi'an, Shaanxi 710004; \\ ${ }^{2}$ Beijing Novartis Pharma Ltd., Beijing 100004; ${ }^{3}$ Department of Public Health, Key Laboratory of Environment and Genes \\ Related to Diseases, Health Science Center, Xi'an Jiaotong University, Xi'an, Shaanxi 710061; ${ }^{4}$ Department of Orthopedics, \\ The First Clinical Medical College, Shaanxi University of Chinese Medicine, Xianyang, Shaanxi 712000, P.R. China
}

Received May 9, 2017; Accepted September 26, 2018

DOI: $10.3892 /$ etm.2018.6843

\begin{abstract}
Phosphoadenosine 5'-phosphosulfate synthetase 2 (PAPSS2) has been shown to be important in the development of normal skeletal structure. The aim of the present study was to evaluate the role of PAPSS2 in the differentiation of chondrocytes as well as their mechanisms. Using RNA interference-mediated via a lentivirus and a retrovirus, PAPSS2 gene silence and overexpression in ATDC5 chondrogenic cells were performed. Chondrocyte differentiation and chondrogenic-related gene markers associated with extracellular matrix formation were noted. The mRNA and protein expression for Wnt $4, \beta$-catenin and SOX9 genes were observed. The PAPSS2 transcript expression levels progressively decline in ATDC5-induced chondrocyte-like cells during differentiation. Silencing of PAPSS2 expression had a significantly attenuating effect on cell differentiation and decreased expression of collagen II and X. In contrast, over-expression of PAPSS2 promoted the differentiation of ATDC5 chondrogenic cells. The mRNA expression levels of Wnt4 and SOX9 decreased significantly in PAPSS2 knock down cells vs. control cells. However, this expression was increased in the cells over-expressing PAPSS2. These data indicate that PAPSS2 regulates aggrecan activity as well as cell differentiation. The findings favor a mechanism by which PAPSS2 induces differentiation in ATDC5 cells via direct regulation of early signaling events that promote formation
\end{abstract}

Correspondence to: Professor Weizhuo Wang, Department of Orthopedic Surgery, The Second Affiliated Hospital, Xi'an Jiaotong University, 157 Xiwulu, Xi'an, Shaanxi 710004, P.R. China

E-mail: wangweizhuo@mail.xjtu.edu.cn

Abbreviations: PAPSS2, 3'-phosphoadenosine 5'-phosphosulfate synthetase 2; RNAi, RNA interference; SEMD, spondyloepimetaphyseal dysplasia; shRNA, small hairpin RNA; GFP, green fluorescent protein; COL2, collagen type II; COLX, collagen type $\mathrm{X}$

Key words: 3'-phosphoadenosine 5'-phosphosulfate synthetase 2, chondrocyte, ATDC 5 , Wnt, $\beta$-catenin, chondroblasts of collagenous matrix components. This control is probably mediated via extracellular matrix formation $\mathrm{Wnt} / \beta$-catenin signaling pathways.

\section{Introduction}

The formation of endochondral bone is a complex developmental process. This process is initiated by the differentiation and subsequent proliferation of mesenchymal stem cells to chondroblasts. After leaving the cell cycle for terminal differentiation, the chondrocytes become pre-hypertrophic and finally hypertrophic. Cytokines, hormones and transcription factors may all affect this process known as chrondrogenesis (1-3).

Sulfate conjugation reactions are catalyzed via sulfotransferase enzymes via 3'-phosphoadenosine 5'-phosphosulfate (PAPS). PAPS serves as a high-energy sulfate donor and is synthesized from adenosine trisphosphate and inorganic sulfate. This synthesis uses two isoforms of PAPS synthetase (PAPSS): PAPSS1 and PAPSS2 (4-7). Availability of PAPS is a prerequisite for the sulfation of biological molecules, including proteoglycans that function as key extracellular matrix components. This highlights the importance of sulfation in bone development and growth, which in turn depends on the integrity of the extracellular matrix. Due to the requirement for PAPS in sulfation, the biosynthesis of PAPS may influence the rate of sulfation reactions in cells.

Previously, a large Pakistani pedigree was reported to have a homozygous mutation (S475X) resulting in spondyloepimetaphyseal dysplasia (SEMD), Pakistani type. The symptoms include enlarged knee joints, short stature, short, bowed lower limbs, as well as kyphoscoliosis and brachydactyly (characterized by complex shortening of the digits) (8-11). SEMD of the Omani type is caused by deficient chondroitin 6-O-sulfotransferase activity due to an abnormal PAPSS2 gene that impairs PAPS biosynthesis $(4,12)$. A deficiency of PAPSS2 activity results in long bone shortening and bowing, as well as knee arthritis and degenerative joint disease. Mutant mice lacking PAPSS2 activity have been proposed as a model to study PAPSS2 deficiency-associated arthrosis, due to the features of premature and degenerative joint disease and other similarities to human SEMD (9). Of note, postnatal skeletal development is specifically affected in this PAPSS2 mutant 
mouse model. Although the skeleton appears to be normal in newborn brachymorphic mice, the columnar and hypertrophic zones of the epiphyseal growth plates are small, which is consistent with reduced growth $(11,13)$.

In previous studies by our group, microarray analysis provided evidence for the involvement of PAPSS2 genes in patients suffering from endemic knee osteoarthritis and Kashin-Beck disease, which manifests as shortened long bones and enlarged joints in the knees and fingers; in addition, it was demonstrated that PAPSS2 influenced osteoblast differentiation via the Smad signaling pathway (14). These observations suggest that PAPSS2 participates in fibrillogenesis and/or matrix calcification/mineralization. However, the precise mechanisms through which PAPSS2 influences cartilage development and formation are still largely elusive. To investigate cell signaling mechanisms involving PAPSS2, biochemical and molecular studies on the structural and functional characteristics of this enzyme are required. The present study examined the role of PAPSS2 in the extracellular signal-regulated kinase pathway that controls chondrocyte differentiation to better understand how PAPSS2 influences chondrogenesis.

\section{Materials and methods}

Ethics statement. The present study was approved by the Animal Experimental Ethics Committee of Xi'an Jiaotong University (Xi'an, China).

Cell line and culture. Experiments were performed using the murine teratoma cell line ATDC5 (American Type Culture Collection, Manassas, VA, USA). They are chondrogenic cells with processes analogous to chondrocyte differentiation $(15,16)$. The cells were cultured on Dulbecco's modified Eagle's medium with nutrient mixture F-12 (DMEM/F-12; Life Sciences; Thermo Fisher Scientific, Inc.) with $10 \%$ fetal bovine serum (FBS; Gibco; Thermo Fisher Scientific, Inc., Waltham, MA, USA) as well as $100 \mathrm{U} / \mathrm{ml}$ penicillin and $100 \mathrm{mg} / \mathrm{ml}$ streptomycin. Cell culture was performed at $37^{\circ} \mathrm{C}$ in a humidified atmosphere with $5 \% \mathrm{CO}_{2}$. The seeding density was $1 \times 10^{4}$ cells $/ \mathrm{cm}^{2}$ and cells were passaged every 5-7 days, but for no more than 20 passages. For pre-chondrocytic ATDC5-cell differentiation, the cells were induced via a chondrogenic growth medium containing $100 \mathrm{mg} / \mathrm{ml}$ ascorbic acid $(17,18)$. To induce chondrocytic differentiation, cells were seeded on 6-well plates and incubated for 2 weeks in DMEM/F-12 supplemented with 1,600 nM human biosynthetic insulin (Sigma-Aldrich; Merck KGaA, Darmstadt, Germany) (18).

PAPSS2 small hairpin (sh)RNA lentivirus packaging, retrovirus vector, assessment of viral titers and cell transfection. A small hairpin RNA (shRNA) lentiviral packaging system (Open Biosystems, Lafayette, CO, USA) was used to modify PAPSS2 gene expression in accordance with the manufacturer's instructions (14). The human embryonic kidney cell line (293T) was obtained from the American Type Culture Collection (Manassas, VA, USA) and transfected with expression constructs (pLenti-P2A or pLenti-P2B) or constructs containing a scrambled shRNA sequence and associated packaging. PAPSS 2 shRNA sequences are presented in
Table I. Five individual pLB-PAPSS2 shRNA (P-S) vectors (each, $500 \mathrm{ng} / \mu \mathrm{l}$ ) and a control pLB-scramble shRNA (PLB) vector (Open Biosystems) were co-transfected with the packaging plasmids (500 ng/ $/ \mathrm{l})$. Retroviral vector pBMP-PAPSS2 was constructed by inserting a full-length $3.64 \mathrm{~kb}$ PAPSS2 cDNA (access no. NM_011864) into the EcoRI and NotI site of pBMN-I-GFP (Addgene) and packaging was performed following the protocol from the Dr Garry Nolan Laboratory, Stanford University (Stanford, CA, USA) (14). Briefly, retrovirus vectors pBMN-I-GFP (control vector) and pBMN-PAPSS2 were separately transfected into the Phoenix-eco tropic packaging cells using the $\mathrm{CaCl}_{2}$ precipitation method. A total of 2-3 days later, the viral supernatant was harvested, and lentiviral titers were assessed in $293 \mathrm{~T}$ cells with serial dilutions of the lentivirus in the presence of $4 \mu \mathrm{g} / \mathrm{ml}$ polybrene (Sigma-Aldrich; Merck KGaA). Following the transfection, the cells were placed in a $32^{\circ} \mathrm{C}$ humidified incubator for $48 \mathrm{~h}\left(32^{\circ} \mathrm{C}\right.$ aids in stabilizing the virus). The media containing infectious virus was harvested and filtered through a $0.45 \mathrm{~mm}$ filter for the titration assay and infecting ATDC5 cells and following $24 \mathrm{~h}$, virus containing media was removed and replaced with fresh complete medium. Following further $48 \mathrm{~h}$, GFP and PAPSS2 protein expression were confirmed by observation of GFP+ cells, immunostaining, and western blot analysis. The retroviruses carrying pBMP-PAPSS2 and pBMN-I-GFP were then used to infect $70-80 \%$ subconfluent ATDC5 cells in the presence of $6 \mu \mathrm{g} / \mathrm{ml}$ polybrene (Sigma-Aldrich; Merck KGaA) and viral supernatant for $24 \mathrm{~h}$. After induction with chondrogenic medium for various durations according to the experimental protocol, the cells were analyzed. On days $0,2,4,6,8$ and 10 , they were then detached from 6-well plates by trypsinization for counting with a cell counting chamber.

Construction of overexpression vectors and production of recombinant retrovirus. The retroviral vector $\mathrm{pBMN-PAPSS} 2$ carried a full-length 3.64-kb PAPSS2 complementary (c)DNA (GenBank accession no. NM_011864). This construct was inserted into the EcoRI and NotI restriction sites of the pBMN-I-green fluorescent protein (GFP) expression plasmid (Addgene, Cambridge, MA, USA). This downstream insertion and viral packaging was performed based on Stanford/Nolan lab protocols (14). In brief, the retroviral pBMN-I-GFP (control) as well as pBMN-PAPSS2 vectors were transfected separately into Phoenix ecotropic packaging cells via calcium chloride precipitation. At $48 \mathrm{~h}$ after transfection, the cells were cultured at $32^{\circ} \mathrm{C}$ for $48 \mathrm{~h}$ to stabilize the virus. The media with infectious viral particles were collected and filtered through a $0.45-\mu \mathrm{m}$ filter to complete titration assays and transfected into ATDC5 cells. The expression of GFP and PAPSS2 was measured by fluorescence detection, immunostaining and western blot analysis. The pBMN-PAPSS2 and pBMN-I-GFP were used to transfect ATDC5 cells in subconfluent culture ( $80 \%$ subconfluency) with $6 \mu \mathrm{g} / \mathrm{ml}$ polybrene. Cell proliferation was assessed in DMEM/F12 in 6-well plates. Cells were induced under conditions to either overexpress or silence the PAPSS2 gene (depending on the differentiation RNA interference medium used). On days $0,2,4,6,8$ and 10, they were then detached from 6 -well plates by trypsinization for counting with a cell counting chamber. 
Table I. Short hairpin RNA sequences used with 3'-phosphoadenosine 5'-phosphosulfate synthetase 2.

Primers $\left(5^{\prime}-3^{\prime}\right)$

\begin{tabular}{|c|c|c|c|}
\hline & & & \\
\hline Target & Sequence $\left(5^{\prime}-3^{\prime}\right)$ & Sense & Antisense \\
\hline TRCN0000353201 & CCGGGCGTGGAAAGTGTTG & GCGTGGAAAGTGTT & ATCTGTCAACAC \\
\hline & ACAGATCTCGAGATCTGT & GACAGAT & TTTCCACGC \\
\hline & CAACACTTTCCACGCTTTTTG & & \\
\hline TRCN0000280504 & CCGGCCATCATGTGAGCAGG & CCATCATGTGAGCA & TTGTTCCTGCT \\
\hline & AACAACTCGAGTTGTTCCTG & GGAACAA & CACATGATGG \\
\hline & CTCACATGATGGTTTTTG & & \\
\hline TRCN0000280502 & CCGGGCAGGAGAGATTAAAG & GCAGGAGAGATTA & AAACCCTTTAAT \\
\hline & GGTTTCTCGAGAAACCCTTT & AAGGGTTT & СTCTCCTGC \\
\hline & AATCTCTCCTGCTTTTTG & & \\
\hline TRCN0000280566 & CCGGGCTCTATTACAGGACCC & CTCTATTACAGGAC & TTCAGGGTCCT \\
\hline & TGAACTCGAGTTCAGGGTCC & CCTGAA & GTAATAGAGC \\
\hline & TGTAATAGAGCTTTTT & & \\
\hline TRCN0000024944 & CCGGGCTTTGGAAGAGTACC & GCTTTGGAAGAGTA & TACAAGGTACT \\
\hline & TTGTACTCGAGTACAAGGT & CCTTGTA & CTTCCAAAGC \\
\hline & АСТCTTCCAAAGCTTTTT & & \\
\hline
\end{tabular}

$R T$-qPCR. Tissues from 14-day-old c57BL/6J female mice (weight, 30 \pm 3 g; Experiment Center, Xi'an Jiaotong University), including lung, spleen, kidney, liver, muscle, heart, calvaria, brain and bone, were collected and homogenized prior to RNA extraction $\left(5 \mathrm{~min} ; 2,000 \mathrm{x} \mathrm{g} ; 4^{\circ} \mathrm{C}\right)$. The total RNA was extracted using TRIzol and the RNA concentration was determined according to spectrophotometry, followed by RT-qPCR (both Invitrogen; Thermo Fisher Scientific, Inc.). An RNeasy Mini kit (Qiagen, Valencia, CA, USA) was used to extract RNA from growth plate chondrocyte cultures. The mRNA levels of aggrecan, Wnt4, $\beta$-catenin, SRY-box (SOX)-9, collagen type II (COL2) and collagen type X (COLX) were then determined as described previously (19). Total RNA $(1 \mu \mathrm{g})$ was reverse transcribed into the cDNA using an Omniscript RT kit (Qiagen). The resulting cDNA was used as a template at 1:100 dilution to measure the relative mRNA levels via real-time qPCR on an ABI Prism 7300 Sequence Detection System (Applied Biosystems; Thermo Fisher Scientific, Inc.). qPCR was based on the SYBR ${ }^{\circledR}$ Green detection method and was performed using the following primers: PAPSS2 forward, 5'-TGTAAAACGACGGCCAGT-3' and reverse, 5'-CAGGAA ACAGCTATGACC-3'; COLX forward, 5'-AGTGCTGTC ATTGATCTCATGGA-3' and reverse, 5'-TCAGAGGAATAG AGACCATTGGATT-3'; SOX9 forward, 5'-AGGAAGCTG GCAGACCAGTA-3' and reverse, 5'-TCCACGAAGGGTCTC TTCTC-3'; COL2A1 forward, 5'-CTACGGTGTCAGGGC CAG-3' and reverse, 5'-GCAAGA-TGAGGGCTTCCATA-3'; Wnt4 forward, 5'-AACCGGCGCTGGAACTG-3' and reverse, 5'-GGTCCCTTGTGTCACCACCTT-3'; $\beta$-catenin forward, 5'-TTTATGAGTGGGAGCAAGGC-3' and reverse, 5'-TGC CCTCATCTAGTGTCTCA-3'; $\beta$-actin forward, 5'-CACCCT GTGCTGCTCACCGAGGCC-3' and reverse, 5'-CCACAC AGATGACTTGAGCTCAGG-3'. Reactions were performed on an ABI The PRISM 7500 sequence detection system with SYBR ${ }^{\circledR}$ GREEN PCR Master Mix (Applied Biosystems; Thermo Fisher Scientific, Inc.) was used according to the manufacturer's instructions. The PCR conditions were $94^{\circ} \mathrm{C}$ for $1 \mathrm{~min}$, followed by $95^{\circ} \mathrm{C}$ for $30 \mathrm{sec}$ and $58^{\circ} \mathrm{C}$ for $40 \mathrm{sec}$ for a total of 35 cycles. All of the reactions were run in triplicate and normalized to the housekeeping gene $\beta$-actin. The relative mRNA expression in each group was calculated using the quantitative cycle method (20).

Western blot analysis. Cells were lysed with Nonidet $\mathrm{P}$ (NP)-40 buffer (1\% NP-40, $0.15 \mathrm{M} \mathrm{NaCl}, 50 \mathrm{mM}$ Tris; $\mathrm{pH}$ 8.0) containing protease inhibitors (Sigma-Aldrich; Merck $\mathrm{KGaA}$ ). A bicinchoninic acid assay was used for protein quantitation (Pierce; Thermo Fisher Scientific, Inc.). Samples were denatured in SDS buffer and $50 \mu \mathrm{g}$ protein was loaded per lane and separated by electrophoresis on SDS gels with $8-10 \%$ polyacrylamide. The proteins were transferred onto polyvinylidene difluoride membranes. These were then incubated with the following antibodies: Anti-PAPSS2 (cat. no. ab37611; 1:100) anti-COL2 (cat. no. ab185430; $2 \mu \mathrm{g} / \mathrm{ml}$ ) and anti-COLX (cat. no. ab58632; $2 \mu \mathrm{g} / \mathrm{ml}$ ), and anti- $\beta$-actin (cat. no. AC-40; 1:2,000; all Abcam, Cambridge, MA, USA) for $12 \mathrm{~h}$ at $4^{\circ} \mathrm{C}$. Subsequently, membranes were washed and incubated with horseradish peroxidase (HRP)-conjugated secondary anti-mouse $[\mathrm{m}-\mathrm{IgG \kappa}$ binding protein horseradish peroxidase (HRP) conjugated; cat. no. sc-516102] or anti-rabbit (mouse anti-rabbit HRP-IgG; cat. no. sc-2357) antibodies (each 1:5,000; Santa Cruz Biotechnology, Inc., Dallas, TX, USA) at room temperature for $2 \mathrm{~h}$. Proteins were visualized using enhanced chemiluminescence (Advansta, Inc., Menlo Park, CA, USA) and the blots were imaged and quantified with the Fluor-S Multi-Imager system and Multi-Analyst software version 1.1 (Bio-Rad Laboratories, Inc., Hercules, CA, USA).

Immunocytochemical and immunohistochemical staining. For immunocytochemical staining, ATDC5 cells $\left(1 \times 10^{4}\right.$ cells/well) were seeded in 24 -well plates containing poly-L-lysine-coated $7 \times 7 \mathrm{~mm}^{2}$ coverslips. These were induced 
with chondrogenic media. At 14 days after seeding, the cells were fixed with $4 \%$ paraformaldehyde in PBS for $20 \mathrm{~min}$ and rinsed with PBS at $4^{\circ} \mathrm{C}$. The avidin-biotin-peroxidase complex method was used for immunocytochemistry. In brief, cells were permeabilized with $0.2 \%$ Triton $\mathrm{X}-100$ for $15 \mathrm{~min}$ at $4^{\circ} \mathrm{C}$ to facilitate uptake of antibodies for labeling of intracellular antigens. Prior to exposure to primary antibodies, unspecific binding was blocked with $10 \%$ FBS in PBS for $1 \mathrm{~h}$ at $37^{\circ} \mathrm{C}$, followed by washing with PBS. Cells were incubated overnight with the primary antibody (PAPSS2; as above) at $4^{\circ} \mathrm{C}$, washed and incubated with HRP-conjugated secondary antibodies (anti-mouse as above) for $1 \mathrm{~h}$ at room temperature, prior to visualization using chemiluminescence (Santa Cruz Biotechnology, Inc., Dallas, TX, USA) according to the manufacturer's instructions. Cells were washed with PBS. A $0.3 \%$ (v/v) 3,3' diaminobenzidine solution in $0.1 \%$ (v/v) hydrogen peroxide was used for visualization via peroxidase reaction. DAB/peroxide was used for visualization of the secondary antibody. For microscopic analysis, the cells were counterstained with hematoxylin, washed with distilled water and successively dehydrated in ethanol (70, 95 and 99.9\%) as well as xylene. Images of the cells were captured using a Nikon light microscope (Nikon, Tokyo, Japan). The negative control, which was prepared without incubation of cells with primary antibody, verified the specificity and reliability of the secondary antibody. Cells with visible yellow staining were considered positively stained.

For immunohistochemical staining, cartilage slices were fixed with $4 \%$ paraformaldehyde for $24 \mathrm{~h}$ at $4^{\circ} \mathrm{C}$ following the removal of the tissue and decalcified at room temperature for $10 \mathrm{~min}$ in $3 \%$ ethylenediaminetetraocetic acid. Samples were dehydrated in a series of alcohol, cleared in xylene, and embedded in paraffin wax at room temperature. Paraffin sections $(6-8 \mu \mathrm{m})$ were cut, mounted on slides, pretreated with $2 \%$ poly-L-lysine at $4^{\circ} \mathrm{C}$ for $10 \mathrm{~min}$ and stored at room temperature until used. Deparaffinized cartilage sections were incubated with testicular hyaluronidase $(2 \mathrm{mg} / \mathrm{ml}$ in PBS, pH 5; cat. no. E0037; Shanghai Baoman Biotechnology Co., Ltd., Shanghai, China) for $30 \mathrm{~min}$ at room temperature. Samples were incubated with primary antibodies (as aforementioned) overnight at $4^{\circ} \mathrm{C}$ and visualized using alkaline phosphatase-labeled secondary antibodies (1:100) obtained form and using the Histostain ${ }^{\mathrm{TM}}$-SAP kit (cat. no. SAP-9100; OriGene Technologies, Inc., Rockville, MD, USA).Visualization was performed for $30 \mathrm{~min}$ at room temperature using 3-hydroxy-2-naphthoic acid 2,4-dimethylanilide (1\%). Finally, nuclei were counterstained with hematoxylin for $2 \mathrm{~min}$ at room temperature. Sections were examined and counted using a light microscope for cytoplasmic and pericellular staining. Four to six randomly selected fields in each zone were counted at a magnification of $\mathrm{x} 400$.

Alcian blue staining. Alcian blue staining of ATDC5 cells was performed as previously described (21). Following fixing in $4 \%$ (w/v) parafomraldehyde in PBS for $30 \mathrm{~min}$, samples were stained with Alcian blue (Sigma-Aldrich; Merck KGaA) for 5 min, followed by dehydration with $95 \%$ ethanol. Images were then captured under a microscope (Nikon TE2000-S; Nikon) and analyzed using Image-Pro Plus 6.0 software (Media Cybernetics, Inc., Rockville, MD, USA).
Statistical analyses. SPSS software (version 17.0; SPSS, Inc., Chicago, IL, USA) was used for one-way analysis of variance, followed by Tukey's honest significant differences test for post-hoc analysis. For comparison between two groups, statistical significance was assessed using Student's t-test. All experiments were performed at least in triplicate. Values are expressed as the mean \pm standard deviation. $\mathrm{P}<0.05$ was considered to indicate a statistically significant difference.

\section{Results}

Expression of PAPSS2 $m R N A$ in cartilage and role in medium-induced cell differentiation. PAPSS 2 mRNA expression was measured in various tissues from mice via RT-qPCR. The expression levels were elevated in calvaria, bone, liver and lung, and moderately in the heart. The expression in calvaria and long bone was significantly higher than in the other tissues as indicated in the figure (Fig. 1A). The PAPSS 2 mRNA expression was low in the muscle, spleen, kidney and brain (Fig. 1A). The housekeeping gene murine $\beta$-actin was present at constant levels across all tissues. This highlights the reliability of the chosen RT-qPCR method in measuring PAPSS2 mRNA. These results are consistent with those of a previous study (4). PAPSS2 mRNA expression was higher in mouse chondrocytes than in other tissues (4). PAPSS2 was also detected in cartilage tissue following immunohistochemical staining for PAPSS2 (Fig. 1A-C), and in the murine ATDC5 cell line at the protein level by western blot analysis (Fig. 1D and E). These results indicate marked PAPSS2 mRNA expression in mouse cartilage, as well as protein expression in mouse chondrocytes exposed to chondrogenic media. The in vitro results indicate an important role for PAPSS2 in chondrocyte differentiation. To analyze the molecular functions of PAPSS 2 in the proliferation and differentiation of chondrocytes, groups of PAPSS2-overexpressing (PO) and -silenced (PS) ATDC5 cells were established (Fig. 2A-D). The silencing efficiency of the RNAi technique was assessed by RT-qPCR (data not shown) and western blot analysis following transfection with siRNA specific to PAPSS2 and PAPSS2 mRNA. The protein expression of PAPSS2 was almost silenced in these stably transfected cells relative to that in the controls (transfected with empty vector), at 7 and 14 days post-transfection. Compared with the parental cells, the PAPSS2 protein had decreased expression in knockdown cells when assessed at 7 days (Fig. 2B and D). At 14 days of differentiation, no morphological alterations were observed in the cells of the PS and PO groups when compared with control ATDC5 cells (Fig. 2E). There were no morphological changes after chondrogenic differentiation compared with baseline (e.g. day 14 vs. 0).

PAPSS2 promotes aggrecan activity and chondrocyte matrix production in chondrogenic terminal differentiation events. During development, chondrocytes undergo a hypertrophic phase followed by terminal differentiation and mineralization. To highlight the importance of PAPSS2 in chondrocyte differentiation, a lentivirus-mediated RNA interference (RNAi) technique was applied to silence the PAPSS2 gene in ATDC5 cells exposed to chondrogenic media. To analyze the effect of PAPSS2 overexpression and knockdown on aggrecan expression, Alcian blue staining assays were performed after induction 
A

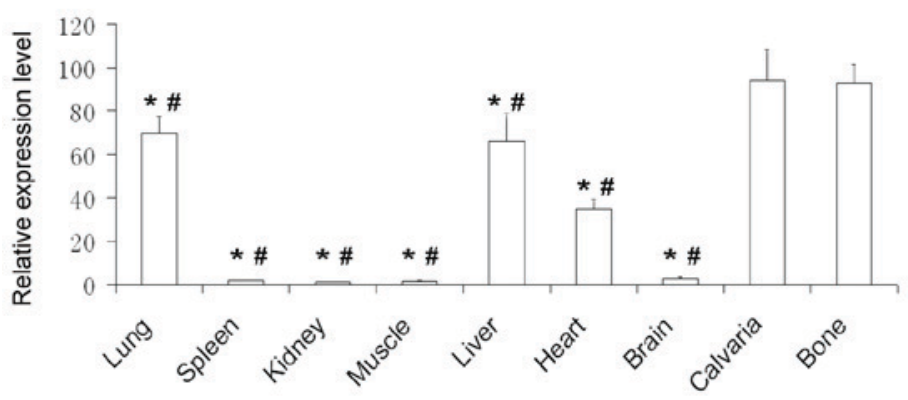

C



PAPSS2

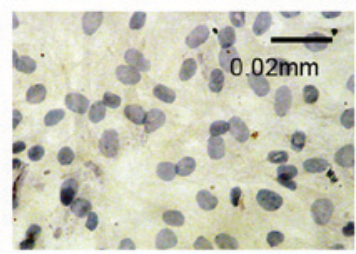

Control
D

B

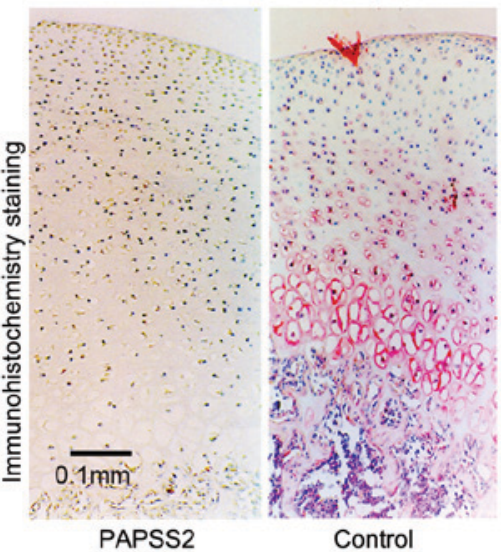

PAPSS2

$\beta$-actin

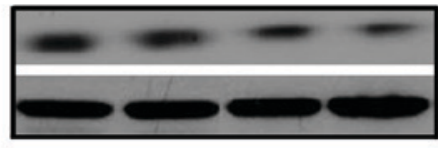

E
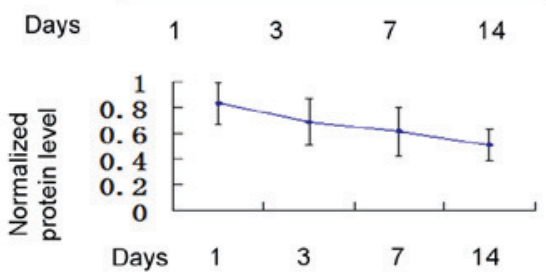

Figure 1.PAPSS2 is present in chondrocytes, bone and osteoblasts. (A) Reverse transcription-quantitative polymerase chain reaction analysis of PAPSS2 mRNA expression in 14-day-old mouse tissues. The total RNA relative to $\beta$-actin expression from calvaria, long bone, brain, heart, liver, muscle, spleen, kidney and lung. (B) Immunohistochemical localization of PAPSS2 cartilage of the knee in adult mice (magnification, x100; scale bar, 0.1 mm). (C) Immunocytochemical staining for PAPSS2 in monolayer chondrocytes (ATDC5 cells at the 12th day; magnification, x400; scale bar, 0.02 mm). Western blot analysis of PAPSS2 protein expression in ATDC5 cells treated with culture medium for 0, 3, 7 and 14 days. (D) Representative western blot image and (E) quantified expression levels normalized to $\beta$-actin, indicating that the results are consistent with those in $B$. Values are expressed as the mean \pm standard deviation ( $=6$ ). ${ }^{*} \mathrm{P}<0.0001$ vs. calvaria; ${ }^{\#} \mathrm{P}<0.0001$ vs. long bone. PAPSS2, 3'-phosphoadenosine 5'-phosphosulfate synthetase 2.

A

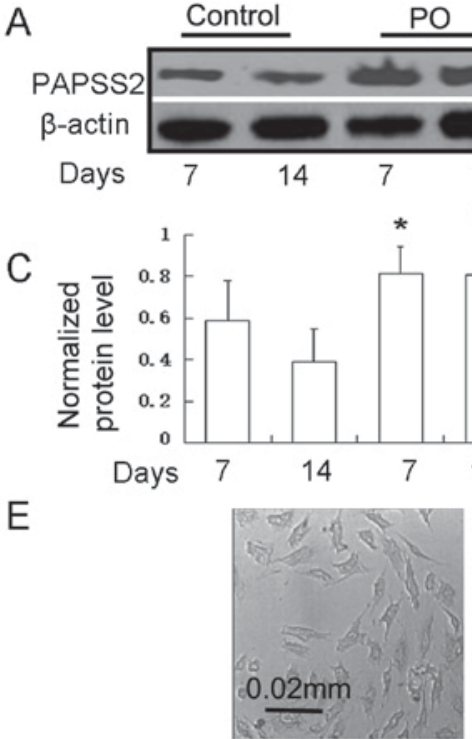

PS
$\mathrm{PO}$

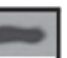

14

$\#$

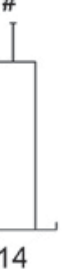

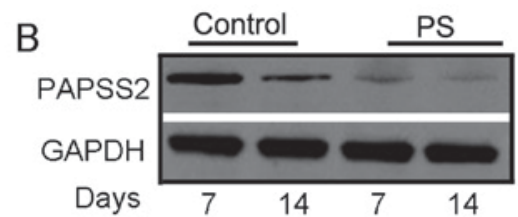

$\mathrm{D}$

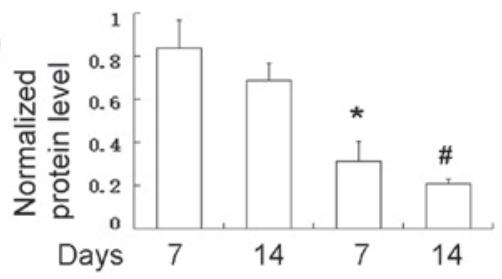



Control

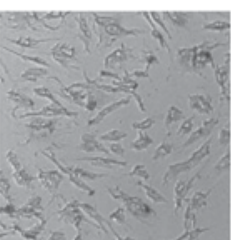

$\mathrm{PO}$

Figure 2. (A) The Phoenix ecotropic packaging cell line was only used for packaging of the plasmids, which contained pBMN-I-GFP (vector-only control) or pBMN-I-GFP-PAPSS2. After transfection for $48 \mathrm{~h}$, the ATDC5 cells were induced with osteogenic induction culture media for 7 or 14 days. The level of PAPSS2 was assessed by western blot analysis. (B) ATDC5 cells were transfected with pLenti-shRNA PAPSS2 or pLenti-scrambled shRNA viruses for $48 \mathrm{~h}$ and treated with osteogenic induction media for 7 and 14 days. (C) Quantified expression values from A normalized to $\beta$-actin levels. PAPSS2 was decreased in control cells and was significantly elevated in cells of the PO group. (D) Quantified expression values from B normalized to GAPDH levels indicated that the protein expression levels of PAPSS2 slowly decreased in control cells and were markedly lower or even undetectable in the PS group. (E) Morphology of the parental ATDC5 cells and ACTD5 cells grown in differentiation media containing either pLenti PAPSS2-shRNA for knockdown or pBMN-PAPSS2 overexpression vector for 14 days (scale bar, $0.02 \mathrm{~mm}$ ). Overexpression or knockdown of PAPSS2 did not significantly alter the appearance of ATDC5 cells. ${ }^{\text {"P }}<0.05$ vs. 7 day control; " $\mathrm{P}<0.05$ vs. 14 day control. PO, PAPSS overexpression group; PS, PAPSS suppression group; PAPSS2, 3'-phosphoadenosine 5'-phosphosulfate synthetase 2; shRNA, small hairpin RNA; GFP, green fluorescence protein; pLenti, lentiviral plasmid. 

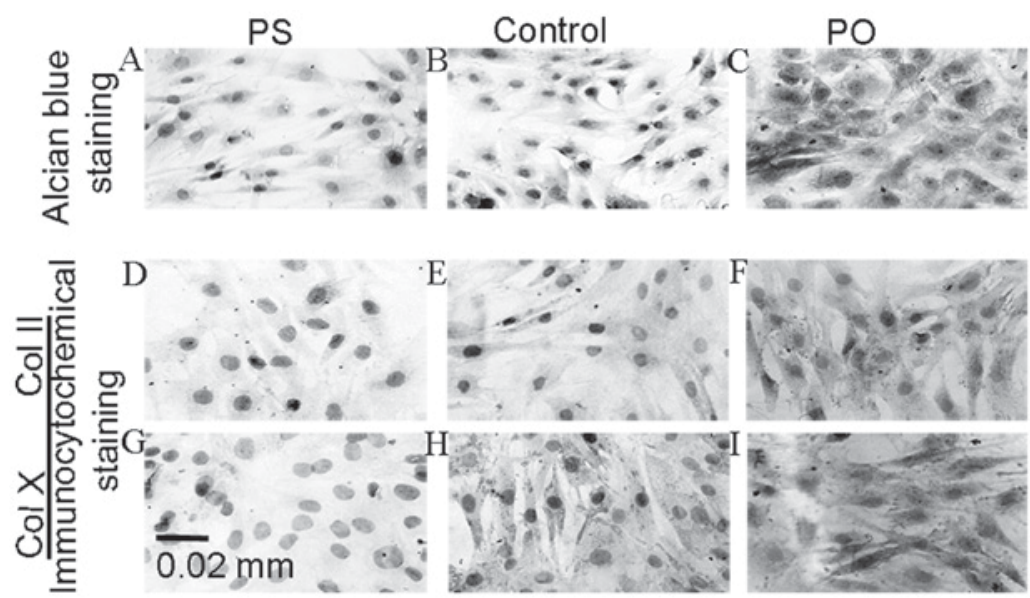

Figure 3. Effect of PAPSS2 expression on extracellular matrix formation. Upon reaching 80\% subconfluency, ACTD5 cells were grown in differentiation media containing either lentiviral plasmid expressing PAPSS2 small hairpin RNA for knockdown or pBMN-PAPSS2 vector for overexpression of PAPSS2 for 14 days. (A-C) Alcian blue staining and immunocytochemical staining for (D-F) Col types II and (G-I) X in the PO, PS and control chondrocytes was performed, respectively (magnification, x400). Col, collagen; PO, PAPSS overexpression group; PS, PAPSS suppression group; PAPSS2, 3'-phosphoadenosine 5 '-phosphosulfate synthetase 2 .

of ATDC5 cells in chondrogenic media containing the vector pBMN-PAPSS2 for 14 days (Fig. 3A). Following the silencing of the PAPSS2 gene, extracellular matrix differentiation was significantly reduced as determined by alcian blue staining and the levels of ColII and COlX were decreased as determined by immunocytochemical staining. To evaluate the effect of PAPSS2 overexpression on differentiation, the ATDC5 cells were induced for 7 days in media either containing retroviral constructs to induce PAPSS2 overexpression or control (empty) constructs. shRNA was used to modify PAPSS2 gene expression. PAPSS2 overexpression resulted in increased levels of ColII and COlX as determined by immunocytochemical staining. Furthermore, extracellular matrix differentiation was significantly increased, as determined by alcian blue staining (Fig. 3A). Of note, PAPSS2 overexpression was associated with marked increases in aggrecan activity and chondrocyte matrix production (Fig. 3A). Furthermore, positive immunostaining for COL2 and COLX was evaluated in the PS and PO groups, revealing that the percentage of the chondrocytes positive for COLX and COL2 in the PS group was significantly lower than that in the control group $(\mathrm{P}<0.01$; Table II) (Fig. 3). The percentage of positive staining for COLX and COL2 in the PO group was significantly higher than that in the controls $(\mathrm{P}<0.001)$.

Effect of ectopic PAPSS2 gene expression on the time course of cell proliferation. The ATDC5 cells upon reaching $80 \%$ subconfluency were grown in chondrogenic media and simultaneously transfected with retroviral pBMN-PAPSS2 vector to induce PAPSS2 overexpression. A lentivirus-mediated RNAi was applied to silence the PAPSS2 gene in ATDC5 cells exposed to chondrogenic media. The effects of these treatments to either overexpress or silence the PAPSS2 gene during ATDC5 proliferation and differentiation were assessed. Cell counts were assessed on days $0,1,2,4,7$ and 10. At the selected time-points, the cells were trypsinized and counted. The cells maintained in medium to overexpress PAPSS2 proliferated at a significantly higher rate compared with the cells in the control group, while proliferation was significantly decreased for cells treated with medium to silence the PAPSS2 gene (Fig. 4).

Effect of ectopic PAPSS2 gene expression on chondrocytespecific markers and modulation of the Wnt/ $\beta$-catenin pathway in the transfected ATDC5 cells. Chondrogenic induction was initiated in ATDC5 cells using chondrogenic media containing retroviral vector pBMN-PAPSS2 (to promote PAPSS2 overexpression) and an empty lentivirus vector (to knockdown PAPSS2 expression) to study the effect of ectopic PAPSS2 gene expression on known chondrogenic markers and collagen proteins. Following exposure to media either containing retroviral constructs to induce PAPSS2 overexpression or control (empty) constructs, the PAPSS2 protein levels were evaluated by western blot analysis. As expected, the levels of various chondrogenic markers were significantly higher in cells incubated in chondrogenic media containing constructs to induce PAPSS2 overexpression vector compared with that in the control group. RT-qPCR analysis indicated significant increases in the expression of the chondrocyte marker genes COLX, COL2, Wnt4, and SOX9 in cells overexpressing PAPSS2 (Fig. 5A-D). However, the levels of various chondrogenic markers were significantly lower in cells of the PAPSS2 silence vector group compared with that in the control group. RT-qPCR analysis indicated significant decreases in the expression of the chondrocyte marker genes COLX, COL2, Wnt4, and SOX9 in cells silenced PAPSS2 (Fig. 5A-D). To further investigate whether PAPSS2 affects Wnt/ $\beta$-catenin signaling expression activation, $\beta$-catenin and Wnt4 levels were observed by RT-qPCR. As presented in Fig. 5, there was a significant increase in Wnt 4 and $\beta$-catenin in the PAPSS2 silence group compared with the control, and a decrease in the PAPSS2 overexpression group compared with the control. These results clearly indicated that both silencing and overexpression of PAPSS2 affected Wnt/ $\beta$-catenin signaling activation. The effect of PAPSS 2 overexpression on COL2 and COLX confirmed the effect observed at the protein level by immunocytochemistry (Fig. 3). In addition, quantification of alcian blue staining after induction of 
Table II. Percentage of positive immunocytochemical staining of chondrocytes of the PS, PO and control groups for types II and $\mathrm{X}$ collagen at 14 days of induction/transfection.

\begin{tabular}{lcc}
\hline Group & $\begin{array}{c}\text { Positive stain } \\
\text { for Col II (\%) }\end{array}$ & $\begin{array}{c}\text { Positive stain } \\
\text { for Col X (\%) }\end{array}$ \\
\hline PS & $22.3 \pm 6.9^{\mathrm{a}}$ & $0.0 \pm 0.0^{\mathrm{b}}$ \\
Control & $55.0 \pm 7.6$ & $44.3 \pm 6.7$ \\
PO & $82.4 \pm 8.0^{\mathrm{a}}$ & $84.7 \pm 6.6^{\mathrm{b}}$ \\
\hline
\end{tabular}

${ }^{a} \mathrm{P}<0.05$ and ${ }^{\mathrm{b}} \mathrm{P}<0.01$ vs. the control group. Values are expressed as the mean \pm standard deviation $(n=8)$. PO, PAPSS overexpression group; PS, PAPSS suppression group; PAPSS2, 3'-phosphoadenosine 5 '-phosphosulfate synthetase 2 ; Col, collagen.

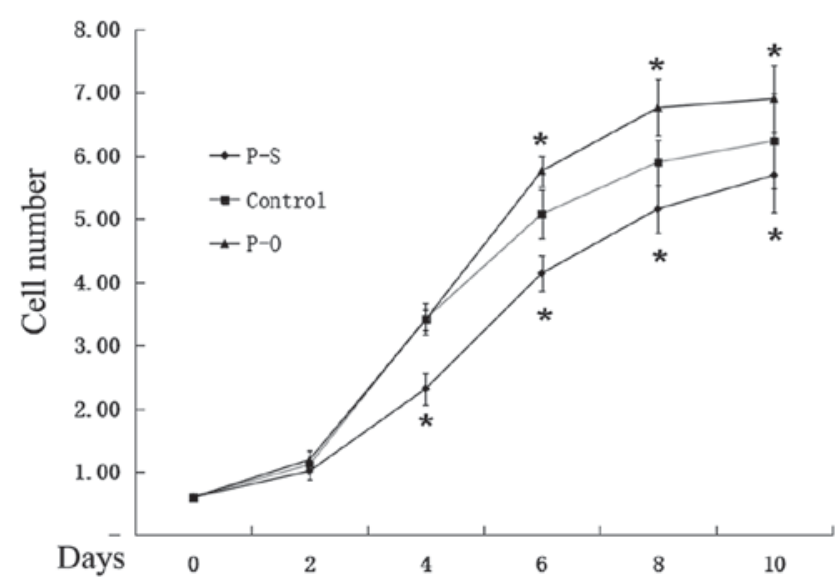

Figure 4. Effect of PAPSS2 expression on the cell proliferation rate. Upon reaching $80 \%$ subconfluency, ATDC5 cells were incubated in differentiation media containing either lentiviral plasmid expressing PAPSS2 smal hairpin RNA for knockdown or pBMN-PAPSS2 vector for overexpression of PAPSS2 for 14 days. Cells were counted on days $0,2,4,6,8$ and 10 . Values are expressed as the mean \pm standard deviation $(n=3)$. " $\mathrm{P}<0.05$ vs. control. PO, PAPSS overexpression group; PS, PAPSS suppression group; PAPSS2, 3'-phosphoadenosine 5 '-phosphosulfate synthetase 2 .

ATDC5 cells in chondrogenic media containing the retroviral vector pBMN-PAPSS2 for 14 days from Fig. 3A indicated that PAPSS2 overexpression was associated with marked increases in aggrecan activity and chondrocyte matrix production (Fig. 5).

\section{Discussion}

PAPSS2 is mostly expressed during the formation of bone elements in the mouse embryo as well as in the cartilage of the newborn mouse $(11,22,23)$. Consistent with these previous studies, the results of the present study indicated that PAPSS 2 is highly expressed in the calvaria and bone tissues of 14-day-old c57BL/6J mice. A notable amount of PAPSS2 was observed in the liver lung and heart, however it was not significant. A minimal amount of PAPSS2 was observed in the brain, muscle, kidneys, spleen. Cartilage is well known to be avascular, alymphatic and aneural, and chondrocytes are its only cell type. Consequently, cartilage tissue has limited innate capacity for self-regeneration (e.g., after damage from physical injury or degenerative disease).
This makes it vulnerable to changing environmental conditions. In addition to physical trauma, autoimmune reactions may lead to dysfunction of cartilage and predispose to severe joint conditions, including osteoarthritis (OA) and rheumatoid arthritis. To achieve a thorough understanding of the factors that regulate cartilage and bone formation, studies are required to identify key signaling pathways and molecules that control chondrogenesis. Chondrogenesis is a key stage in cartilage and bone formation and is the process through which mesenchymal cells differentiate into chondrocytes. In recent decades, interest in the development of novel tissue engineering strategies for cartilage repair has increased, as reflected by the large number of studies performed in this field (24-26).

To improve treatment of cartilage-associated diseases, the potential utility of strategies to regenerate functional cartilage tissue via the induction of chondrogenesis is becoming increasingly important. The chondrogenic ATDC5 cell line is derived from mouse teratocarcinoma cells and enters a sequential differentiation process analogous to that in chondrocytes. This makes them useful for in vitro studies of cell behavior during chondrogenesis. It also provides a useful model for studies addressing the role of signaling pathways in skeletal development and for the characterization of interactions of chondrocytes with novel synthetic materials. To date, $>200$ studies based on the ATDC5 cell line have generated a wealth of data (27-32).

Mutations that inactivate the PAPSS2 gene are associated with severe inherited developmental skeletal disorders, including Kashin-Beck disease, SEMD in humans, and brachymorphism in mice $(14,22,33)$. Under-sulfation due to the inhibition of PAPS synthetase controls extracellular matrix (ECM) expression during chondrogenesis (34-37). PAPSS2 deficiency produces osteochondrodysplasias. These are genetically heterogeneous disorders that damage normal skeletal growth, linear growth, as well as cartilage and bone health. The physical presentation includes a short limb stature, cleft palate, generalized dysplasia and hitchhiker's thumb. Inbred mice display a distinct form of SEMD that is inherited through a recessive pattern attributed to a PAPSS2 polymorphism in 10q23-24. Diastrophic dysplasia is an autosomal recessive osteochondrodysplasia (38-40). It is caused by reduced levels of intracellular sulfate levels, which lead to under-sulfation of proteoglycans. Sequence polymorphisms in the PAPSS2 gene in OA patients were investigated by Oostdijk et al (22). $\mathrm{OA}$ is a musculoskeletal disorder featuring degeneration of articular cartilage. Single nucleotide polymorphism analysis in certain Japanese populations with OA affecting the knee has also provided evidence for involvement of the gene in OA pathogenesis; these investigations revealed differences in the distribution of two PAPSS2 isoforms that were only apparent in OA-affected tissues $(10,11)$.

ATDC5 pre-chondrocytes undergo differentiation into chondrocytes that produce ECM components. The ATDC5 cell line is frequently used to study particular genes in chondrocyte differentiation. In the present study, overexpression of PAPSS2 or knockdown via shRNA suggested an important role for PAPSS2 in initiating differentiation of chondrocytes. After silencing of the PAPSS2 gene, the levels of multiple markers associated with ATDC5 cell differentiation were significantly reduced. PAPSS2 overexpression caused the 

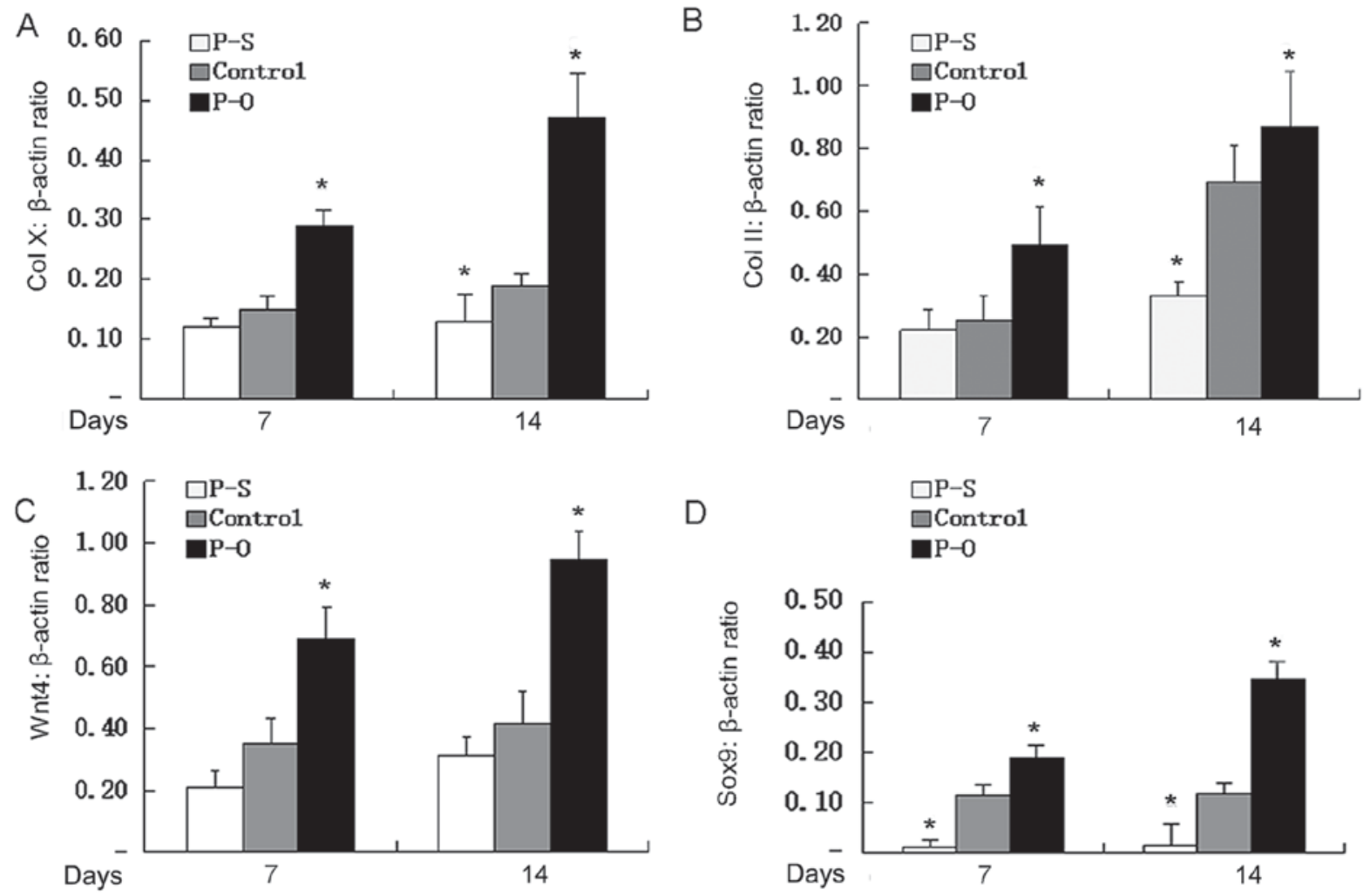

$\mathrm{E}$
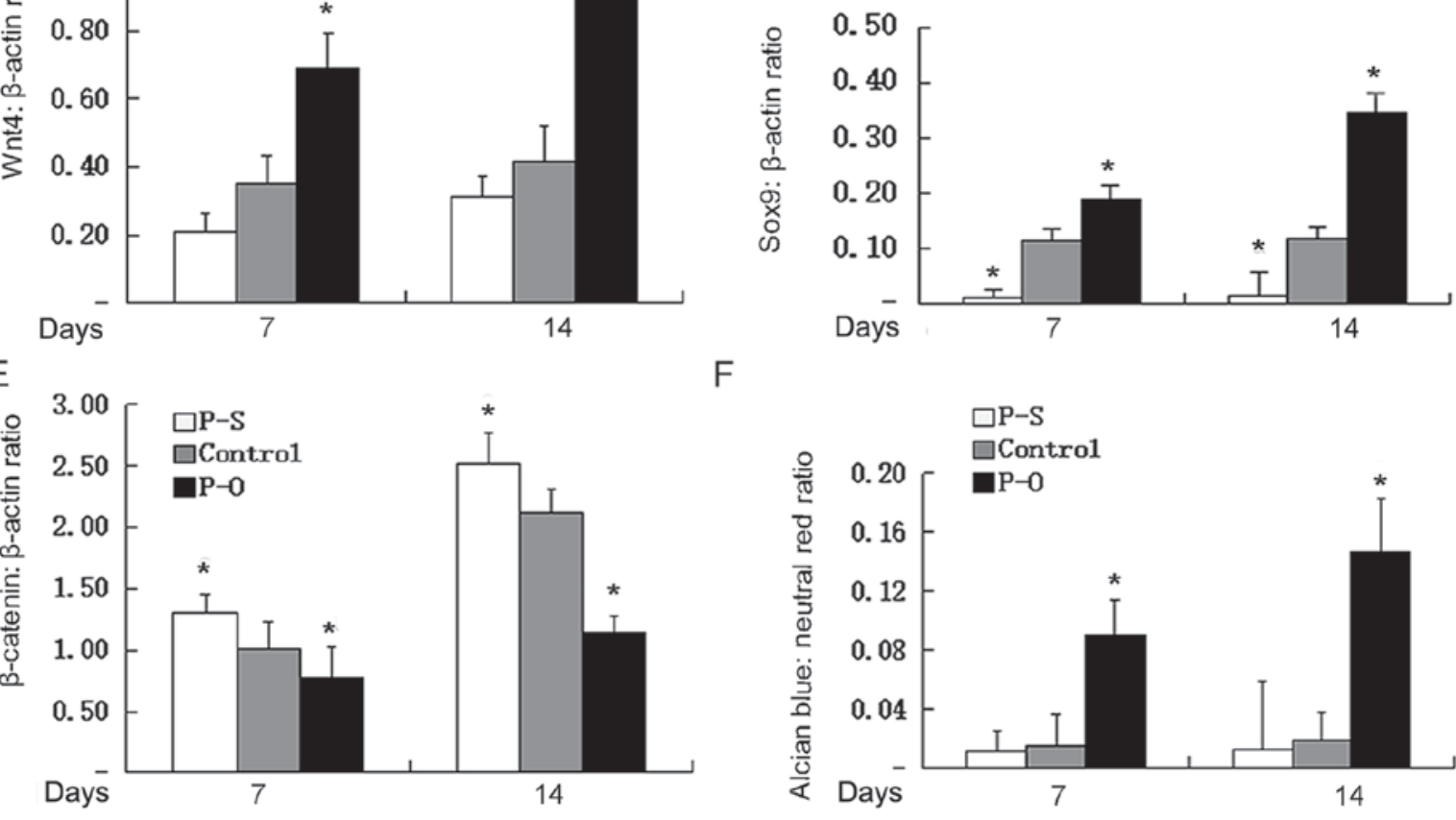

$\mathrm{F}$

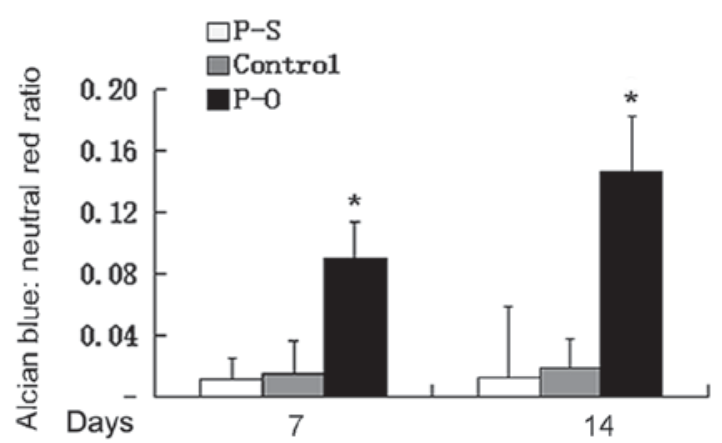

Figure 5. Effect of ectopic PAPSS2 gene expression on chondrogenic genes. Upon reaching 80\% subconfluency, ATDC5 cells were incubated in differentiation media containing either lentiviral plasmid expressing PAPSS2 small hairpin RNA for knockdown or pBMN-PAPSS2 vector for overexpression of PAPSS2 for 7 or 14 days. The expression levels of (A) COLX, (B) COL2, (C) Vnt4, (D) SOX9 and (E) $\beta$-catenin relative to $\beta$-actin expression were determined by reverse transcription-quantitative polymerase chain reaction analysis. (F) Cells were subjected to Alcian blue and neutral red staining, followed by dye extraction for absorbance measurements. The ratio of Alcian blue to neutral red was determined. Values are expressed as the mean \pm standard deviation (n=3). "P<0.05 vs. control. PO, PAPSS overexpression group; PS, PAPSS suppression group; PAPSS2, 3'-phosphoadenosine 5'-phosphosulfate synthetase 2; COL, collagen; SOX, SRY-box.

levels of ColII, COlX, Sox9 and Wint4 to be increased. According to the present immunocytochemistry results, the percentages of chondrocytes positive for COL2 and COLX were significantly higher in the PAPSS2 overexpression group than in the controls. Conversely, the percentages of chondrocytes positive for COL2 and COLX were significantly lower in PAPSS2 knockdown cells compared with those in the control cells.

For chondrogenesis to proceed effectively, differentiation requires specification and maintenance of lineage decisions through activation of stage-specific markers. Genes belonging to the SOX family, which includes $>30$ members, has a central role in regulating chondrogenesis. The SOX9 gene is expressed in mesenchymal precursors and developing chondrocytes until the pre-hypertrophic stage, but not in subsequent lineages.
SOX9 is required for chondrocyte specification and early differentiation (41-43). It maintains growth plate chondrocyte proliferation, delays pre-hypertrophy and facilitates subsequent hypertrophy prior to terminal maturation and apoptosis. In addition, SOX9 directly activates all major cartilage-specific ECM genes expressed by early-stage chondrocytes and is involved in the chondrocyte differentiation pathway at multiple stages. SOX9 has been demonstrated to induce COL2A1 expression by activating a 48 -bp enhancer element residing in the first intron of this gene. It promotes the differentiation of mesenchymal stem cells into chondrocytes (44). SOX9 inactivation in the growth plate resulted in dwarfism due to shortening of columnar and hypertrophic zones and in advanced ossification due to premature pre-hypertrophy and matrix mineralization. These effects are typical of campomelic dysplasia, a severe 
genetic disorder that affects the development of the skeleton, and are consistent with the notion that this disease arises due to growth plate and primordial cartilage defects.

The Wnt/ $\beta$-catenin signaling pathway has an important role in regulating the growth and differentiation of chondrocyte cells (45-47). To study the possible role of PAPSS2 in the regulation of the Wnt4 expression as part of the Wnt pathway, ATDC5 cells were treated with differentiation medium containing PAPSS2 overexpression or shRNA vector. The results indicated that PAPSS 2 treatment inhibits Wnt/ $\beta$-catenin activity and chondrocyte differentiation by upregulating Wnt4 and decreasing $\beta$-catenin mRNA levels in ATDC5 cells. These results suggest that PASS2 suppresses chondrocyte dedifferentiation locally by modulating $\mathrm{Wnt} / \beta$-catenin signaling activity. It may interact with parathyroid hormone-related peptide in a negative feedback loop, and $\mathrm{Wnt} / \beta$-catenin regulates chondrocyte differentiation and initiation of the hypertrophic phase. Wnt/ $\beta$-catenin in chondrocytes is regulated by several factors $(47,48)$. The present study indicated that PAPSS2 regulates Wnt/ $\beta$-catenin signaling expression during the transition of chondrocytes from proliferation to hypertrophic differentiation. The effects of PAPSS 2 on Wnt/ $\beta$-catenin signaling pathways were further examined and silencing as well as overexpression of PAPSS2 affected this signaling pathway. It is suggested that PAPSS2 is an upstream regulator of $\mathrm{Wnt} / \beta$-catenin and its chondrogenic properties may be mediated through mechanisms that need further investigation.

The results of the present study rule out the possibility that PAPSS2 modulates osteopontin activity (either directly or indirectly) or chondrogenic genes via multiple mechanisms, including the Wnt/ $\beta$-catenin signaling pathway. Cells maintained in medium to overexpress PAPSS2 proliferated at a significantly higher rate compared with the cells in the control group, while proliferation was significantly decreased for cells treated with medium to silence the PAPSS2 gene. PAPSS2 overexpression caused the levels of ColII, COIX, Sox 9 and Wint 4 to be increased. After silencing of the PAPSS2 gene, the levels of multiple markers ColII, COlX, Sox 9 and Wint4 associated with ATDC5 cell differentiation were significantly reduced. The present study supports an essential role for PAPSS2 in chondrocyte differentiation via inducing early signaling events. More detailed studies are required to assess other genes identified to be affected by PAPSS2 knockdown, including transcription factors, chondrocyte differentiation enzymes, proteins associated with bone morphogenesis as well as ECM proteins, and their potential mechanisms regarding PAPSS2.

The present results indicate that PAPSS2 induces the chondrogenic differentiation of ATDC5 cells by crosstalk with Wnt signaling. PAPSS2 promotes ATDC5 differentiation by upregulating the production of collagenous matrix components. $\mathrm{Wnt} / \beta$-catenin pathway activation after matrix formation leads to chondrocyte differentiation. Future studies will assess the key markers in the underlying pathways of bone formation or metabolic processes at the gene and protein levels, including the role of PAPSS 2 in the detailed regulation of collagen assembly and its functional properties in Wnt/ $\beta$-catenin signaling pathways during bone and cartilage formation. Transcription and growth factors that regulate expression at the gene and protein level, accumulation and degradation of PAPSS2 will be assessed.
Finally, the role of PAPSS2 in controlling the activity of other signaling pathways will also be addressed.

\section{Acknowledgements}

The authors thank Dr Li Fang and Dr Cao Rui for their critical reading of the manuscript and Dr. He Xiaoning for his technical assistance with fluorescence microscopy and image analysis.

\section{Funding}

This project was funded by the Natural Science Foundation of China (grant no. 81001225), the Fundamental Research Funds for the Central Universities (grant no. xjj2016107), the International Co-operative Plan of Shaanxi (grant no. S2016YFKW0013), the International Co-operative Fund of Xian Jiaotong University (grant no. 08143004) and the Integrative Medicine Research and Innovation Team of Degenerative Bone Disease Prevention of Shaanxi Traditional Chinese Medicine College (grant no. 2013KCT-26).

\section{Availability of data and materials}

The datasets used and/or analyzed during the current study are available from the corresponding author on reasonable request.

\section{Authors' contributions}

WW and XG conceived and designed the experiments. LF performed the experiments. YH analyzed the data. JH and PY helped perform the experiments. WW and LF prepared the manuscript. All authors read and approved the final version of the manuscript.

\section{Ethics approval and consent to participate}

The present study was approved by the Animal Experimental Ethics Committee of Xi'an Jiaotong University.

\section{Patient consent for publication}

Not applicable.

\section{Competing interests}

The authors confirm that they have no competing interests.

\section{References}

1. Stupina TA and Shchudlo MM: A method for making preparations from nondecalcified articular cartilage with sublying subchondral bone for multipurpose studies. Bull Exp Biol Med 157: 401-403, 2014.

2. Mobasheri A, Kalamegam G, Musumeci G and Batt ME: Chondrocyte and mesenchymal stem cell-based therapies for cartilage repair in osteoarthritis and related orthopaedic conditions. Maturitas 78: 188-198, 2014.

3. Acharya C, Yik JH, Kishore A, Van Dinh V, Di Cesare PE and Haudenschild DR: Cartilage oligomeric matrix protein and its binding partners in the cartilage extracellular matrix: Interaction, regulation and role in chondrogenesis. Matrix Biol 37: 102-111, 2014. 
4. Zhang F, Guo X, Wang W, Wu S, Ma W and Yan H: Expression profile analysis of mycotoxin-related genes in cartilage with endemic osteochondropathy Kashin-Beck Disease. BMC Musculoskelet Disord 13: 130, 2012.

5. Xu ZH, Freimuth RR, Eckloff B, Wieben E and Weinshilboum RM: Human 3'-phosphoadenosine 5'-phosphosulfate synthetase 2 (PAPSS2) pharmacogenetics: Gene resequencing, genetic polymorphisms and functional characterization of variant allozymes. Pharmacogenetics 12: 11-21, 2002.

6. Xu ZH, Thomae BA, Eckloff BW, Wieben ED and Weinshilboum RM. Pharmacogenetics of human 3'-phosphoadenosine 5'-phosphosulfate synthetase 1 (PAPSS1): gene resequencing, sequence variation, and functional genomics. Biochem Pharmacol 65: 1787-1796, 2003

7. Peitzmeier SM, Reisner SL, Harigopal P and Potter J: Female-to-male patients have high prevalence of unsatisfactory Paps compared to non-transgender females: Implications for cervical cancer screening. J Gen Intern Med 29: 778-784, 2014.

8. Xu ZH, Otterness DM, Freimuth RR, Carlini EJ, Wood TC, Mitchell S, Moon E, Kim UJ, Xu JP, Siciliano MJ and Weinshilboum RM: Human 3'-phosphoadenosine 5'-phosphosulfate synthetase 1 (PAPSS1) and PAPSS2: Gene cloning, characterization and chromosomal localization. Biochem Biophys Res Commun 268: 437-444, 2000.

9. Ford-Hutchinson AF, Ali Z, Seerattan RA, Cooper DM, Hallgrimsson B, Salo PT and Jirik FR: Degenerative knee joint disease in mice lacking 3'-phosphoadenosine 5'-phosphosulfate synthetase 2 (Papss2) activity: A putative model of human PAPSS2 deficiency-associated arthrosis. Osteoarthritis Cartilage 13: 418-425, 2005.

10. Ramaswamy G, Sohn P, Eberhardt A and Serra R: Altered responsiveness to TGF- $\beta$ results in reduced Papss 2 expression and alterations in the biomechanical properties of mouse articular cartilage. Arthritis Res Ther 14: R49, 2012.

11. Stelzer C, Brimmer A, Hermanns P, Zabel B and Dietz UH Expression profile of Papss2 (3'-phosphoadenosine 5'-phosphosulfate synthase 2) during cartilage formation and skeleta development in the mouse embryo. Dev Dyn 236: 1313-1318, 2007.

12. Thiele H, Sakano M, Kitagawa H, Sugahara K, Rajab A, Höhne W, Ritter H, Leschik G, Nürnberg P and Mundlos S: Loss of chondroitin 6-O-sulfotransferase-1 function results in severe human chondrodysplasia with progressive spinal involvement. Proc Natl Acad Sci USA 101: 10155-10160, 2004.

13. Alnouti $Y$ and Klaassen CD: Tissue distribution and ontogeny of sulfotransferase enzymes in mice. Toxicol Sci 93: 242-255, 2006.

14. Wang W, Li F, Wang K, Cheng B and Guo X: PAPSS2 promotes alkaline phosphates activity and mineralization of osteoblastic MC3T3-E1 cells by crosstalk and Smads signal pathways. PLoS One 7: e43475, 2012.

15. Meng F, He A, Zhang Z, Zhang Z, Lin Z, Yang Z, Long Y, Wu G, Kang Y and Liao W: Chondrogenic differentiation of ATDC5 and hMSCs could be induced by a novel scaffold-tricalcium phosphate-collagen-hyaluronan without any exogenous growth factors in vitro. J Biomed Mater Res A 102: 2725-2735, 2014.

16. Yao Y and Wang Y: ATDC5: An excellent in vitro model cell line for skeletal development. J Cell Biochem 114: 1223-1229, 2013.

17. Newton PT, Staines KA, Spevak L, Boskey AL, Teixeira CC Macrae VE, Canfield AE and Farquharson C: Chondrogenic ATDC5 cells: An optimised model for rapid and physiological matrix mineralisation. Int J Mol Med 30: 1187-1193, 2012.

18. Temu TM, Wu KY, Gruppuso PA and Phornphutkul C: The mechanism of ascorbic acid-induced differentiation of ATDC5 chondrogenic cells. Am J Physiol Endocrinol Metab 299: E325-E334, 2010.

19. Wang WZ, Guo X, Duan C, Ma WJ, Zhang YG, Xu P, Gao ZQ, Wang ZF, Yan H, Zhang YF, et al: Comparative analysis of gene expression profiles between the normal human cartilage and the one with endemic osteoarthritis. Osteoarthritis Cartilage 17: $83-90,2009$

20. Livak KJ and Schmittgen TD: Analysis of relative gene expression data using real-time quantitative PCR and the 2(-Delta Delta C(T)) method. Methods 25: 402-408, 2001

21. Young AD, Phipps DE and Astroff AB: Large-scale double-staining of rat fetal skeletons using Alizarin Red S and alcian blue. Teratology 61: 273-276, 2000.
22. Oostdijk W, Idkowiak J, Mueller JW, House PJ, Taylor AE, O'Reilly MW, Hughes BA, de Vries MC, Kant SG, Santen GW, et al: PAPSS2 deficiency causes androgen excess via impaired DHEA sulfation-in vitro and in vivo studies in a family harboring two novel PAPSS2 mutations. J Clin Endocrinol Metab 100: E672-E680, 2015.

23. Ikeda T, Mabuchi A, Fukuda A, Hiraoka H, Kawakami A, Yamamoto S, Machida H, Takatori Y, Kawaguchi H, Nakamura K and Ikegawa S: Identification of sequence polymorphisms in two sulfation-related genes, PAPSS2 and SLC26A2, and an association analysis with knee osteoarthritis. J Hum Genet 46: 538-543, 2001.

24. Chang CH, Chen CC, Liao CH, Lin FH, Hsu YM and Fang HW: Human acellular cartilage matrix powders as a biological scaffold for cartilage tissue engineering with synovium-derived mesenchymal stem cells. J Biomed Mater Res A 102: 2248-2257, 2014.

25. Van Assche D, Staes F, Van Caspel D, Vanlauwe J, Bellemans J, Saris DB and Luyten FP: Autologous chondrocyte implantation versus microfracture for knee cartilage injury: A prospective randomized trial, with 2-year follow-up. Knee Surg Sports Traumatol Arthrosc 18: 486-495, 2010.

26. Edwards PK, Ebert JR, Janes GC, Wood D, Fallon M and Ackland T: Arthroscopic versus open matrix-induced autologous chondrocyte implantation: Results and implications for rehabilitation. J Sport Rehabil 23: 203-215, 2014.

27. Temu TM, Phornphutkul C and Gruppuso P: Characterization of chondrocyte differentiation of ATDC5 cell line induced by Ascorbic acid. FASEB J 24, 2010.

28. Takayama Y and Mizumachi K: Inhibitory effect of lactoferrin on hypertrophic differentiation of ATDC5 mouse chondroprogenitor cells. Biometals 23: 477-484, 2010.

29. Kwon HJ, Yasuda K, Ohmiya Y, Honma K, Chen YM and Gong JP: In vitro differentiation of chondrogenic ATDC5 cells is enhanced by culturing on synthetic hydrogels with various charge densities. Acta Biomater 6: 494-501, 2010.

30. Itoh R, Miura S, Takimoto A, Kondo S, Sano H and Hiraki Y: Stimulatory actions of lysophosphatidic acid on mouse ATDC5 chondroprogenitor cells. J Bone Miner Metab 28: 659-671, 2010.

31. Challa TD, Rais Y and Ornan EM: Effect of adiponectin on ATDC5 proliferation, differentiation and signaling pathways. Mol Cell Endocrinol 323: 282-291, 2010.

32. Nakatani S, Mano H, Im R, Shimizu J and Wada M: Glucosamine regulates differentiation of a chondrogenic cell line, ATDC5. Biol Pharm Bull 30: 433-438, 2007.

33. Cortes M, Singh B, Kurima K and Schwartz N: PAPS synthetase gene expression relates directly to the murine Brachymorphism (Bm) phenotype. FASEB J 17: A1303-A1303, 2003.

34. Al Attia HM: HLA tissue typing in patients with PAPS (Hughes syndrome) evolving into SLE. Lupus 11: 399-400, 2002.

35. Shimizu C, Lee YC, Fuda H and Strott CA: Promoter analysis of human PAPS synthase (PAPSS) 1 and 2. FASEB J 15: A882-A882, 2001.

36. Gomez JA, Martin H, Amigo MC, Aguirre MA, Cuadrado MJ, Khamashta MA and Hughes GRV: Long-term follow-up in 90 patients with primary antophospholipid syndrome (PAPS). Do they develop lupus? Arthritis Rheum-Us 44: S146, 2001.

37. Venkatachalam KV.Human 3'-phosphoadenosine 5'-phosphosulfate (PAPS) synthase: biochemistry, molecular biology and genetic deficiency. IUBMB Life 55: 1-11, 2003.

38. Weber M, deBandt M, Hayem G, Roux S, Kahn MF and Meyer O: Familial study of primary (PAPS) and secondary (SAPS) syndrome. Arthritis Rheum-Us 40: 1624, 1997.

39. Krakow D, Sebald ET, Pogue R, Rimoin LP, King L and Cohn DH: Analysis of clones from a human cartilage cDNA library provides insight into chondrocyte gene expression and identifies novel candidate genes for the osteochondrodysplasias. Mol Genet Metab 79: 34-42, 2003.

40. Krakow D, Sebald ET, King LM and Cohn DH: Cartilage expressed sequence tags provide insight into chondrocyte gene expression and identify novel candidate genes for the osteochondrodysplasias. Am J Hum Genet 67: 370-370, 2000.

41. Zhu Z, Yu A, Hou M, Xie X and Li P: Effects of Sox9 gene therapy on the healing of bone-tendon junction: An experimental study. Indian J Orthop 48: 88-95, 2014.

42. Raspopovic J, Marcon L, Russo L and Sharpe J: Modeling digits. Digit patterning is controlled by a Bmp-Sox9-Wnt turing network modulated by morphogen gradients. Science 345 : 566-570, 2014 
43. Raspaglio G, Petrillo M, Martinelli E, Puma DDL, Mariani M, De Donato M, Filippetti F, Mozzetti S, Prislei S, Zannoni GF, et al: Sox9 and Hif- $2 \alpha$ regulate TUBB3 gene expression and affect ovarian cancer aggressiveness. Gene 542: 173-181, 2014.

44. Zhang H, Zhao X, Zhang Z, Chen W and Zhang X: An immunohistochemistry study of Sox9, Runx2, and Osterix expression in the mandibular cartilages of newborn mouse. Biomed Res Int 2013: 265380, 2013

45. Yano F, Kugimiya F, Ohba S, Ikeda T, Chikuda H, Ogasawara T, Ogata N, Takato T, Nakamura K, Kawaguchi H and Chung UI: The canonical Wnt signaling pathway promotes chondrocyte differentiation in a Sox9-dependent manner. Biochem Biophys Res Commun 333: 1300-1308, 2005.

46. Yates KE, Shortkroff S and Reish RG: Wnt influence on chondrocyte differentiation and cartilage function. DNA Cell Biol 24: 446-457, 2005.
47. Gao Y, Liu S, Huang J, Guo W, Chen J, Zhang L, Zhao B, Peng J, Wang A, Wang Y, et al: The ECM-cell interaction of cartilage extracellular matrix on chondrocytes. Biomed Res Int 2014: 648459, 2014.

48. Dao DY, Jonason JH, Zhang YC, Hsu W, Chen D, Hilton MJ and O'Keefe RJ: Cartilage-specific $\beta$-catenin signaling regulates chondrocyte maturation, generation of ossification centers, and perichondrial bone formation during skeletal development. J Bone Miner Res 27: 1680-1694, 2012.

This work is licensed under a Creative Commons Attribution-NonCommercial-NoDerivatives 4.0 International (CC BY-NC-ND 4.0) License. 